Mots. Les langages du politique

\title{
Politiques linguistiques au Sénégal au lendemain de l'Indépendance. Entre idéologie et réalisme politique
}

La política lingüística en Senegal después de la Independencia. Entre ideología y realismo político

Language policy in Senegal after Independence. Between ideology and political realism

\section{El Hadji Abdou Aziz Faty}

\section{OpenEdition}

Journals

Édition électronique

URL : https://journals.openedition.org/mots/21747

DOI : $10.4000 /$ mots. 21747

ISSN : 1960-6001

Éditeur

ENS Éditions

Édition imprimée

Date de publication : 31 décembre 2014

Pagination : 13-26

ISBN : 978-2-84788-544-6

ISSN : 0243-6450

\section{Référence électronique}

El Hadji Abdou Aziz Faty, « Politiques linguistiques au Sénégal au lendemain de l'Indépendance. Entre idéologie et réalisme politique », Mots. Les langages du politique [En ligne], 106 | 2014, mis en ligne le 31 décembre 2016, consulté le 22 avril 2022. URL : http://journals.openedition.org/mots/21747 ; DOI : https://doi.org/10.4000/mots. 21747 


\section{Politiques linguistiques au Sénégal au lendemain de l'Indépendance. Entre idéologie et réalisme politique}

L'objectif principal de cet article est de porter un regard sur les conséquences glottopolitiques (Guespin, Marcellesi, 1986) de la planification linguistique dite de francisation héritée du système colonial français. Plus précisément, nous essaierons de répondre à la question suivante : en quoi la perpétuation, par l'État postcolonial sénégalais, de cette politique de francisation a-t-elle, au lendemain de l'Indépendance, impulsé une dynamique politique débouchant sur un engagement sans faille pour la défense des langues nationales? En effet, le choix d'une politique linguistique en faveur de la langue française (langue officielle) et au détriment des langues sénégalaises, reléguées au statut flou et symbolique de «langues nationales», a provoqué une levée de boucliers chez certains acteurs politiques qui continuaient de considérer le français comme une langue impérialiste et de domination. Concrètement, cela s'est traduit par la mise en place d'un certain nombre de plans d'action en vue d'une (re)vitalisation et d'une émancipation des langues nationales, notamment le wolof. Ainsi a-t-on a vu naître des productions littéraires, des dictionnaires, des traductions d'œuvres majeures de la littérature occidentale dans les langues nationales, etc. Ce travail de normativisation linguistique (Aracil, 1965) va de pair avec un engagement pour l'introduction des langues nationales dans le circuit formel de l'enseignement, où le français reste la seule langue d'enseignement. Ainsi cette période se caractérise-t-elle par une lutte acharnée entre deux camps. Le premier, conservateur, tenant du pouvoir, opte pour la francisation tandis que l'autre - les opposants, composés de communistes, d'antiimpérialistes et de nationalistes - plébiscite les langues nationales. Il conviendra ici de rendre compte et d'analyser les différentes idéologies linguistiques mobilisées de part et d'autre en guise d'argumentation et de justification. 


\section{Le français au service du projet colonial?}

Pour comprendre la politique linguistique mise en place par les Français dans leurs colonies, il convient de revenir sur celle qu'ils ont appliquée pendant la Troisième République envers la paysannerie en France métropolitaine. En effet, plusieurs études, dont celle d’Eugen Weber (1983), spécialiste de la France rurale, ont montré que les paysans français qui s'établissaient en dehors de "l'île de Paris» n'étaient pas considérés comme des citoyens à part entière, de par la rusticité de leur langue maternelle. Le livre de Weber, intitulé La fin des terroirs. La modernisation de la France rurale, 1870-1914, dont le titre original en anglais, Peasants into Frenchmen ${ }^{1}$, est plus significatif - la préposition performative et active into induisant le fait de devenir ou se transformeraide à comprendre le processus marquant le passage ou l'intégration d'une partie de la France, paysanne et rurale, à une France moderne et «civilisée » et ce, par le biais de la langue. L'intérêt de l'ouvrage de Weber est d'avoir montré qu'au début de la Troisième République, la langue française se présentait comme une langue étrangère aux yeux de bon nombre de Français, et d'avoir exposé les détails d'une politique de francisation. L'objectif n'est pas ici de retracer l'histoire de la Troisième République dans sa globalité, mais de montrer comment la France est arrivée à mettre sur pied tout un arsenal juridicopolitique et technologique pour imposer la langue française à « près d'un quart de sa population» (Weber, 1983, p.93), à savoir les paysans, afin de pouvoir faire émerger chez eux une conscience d'appartenance à la Nation française. C'est ce que Nancy Kwang Johnson a appelé le processus de «fabrication des Français» (Kwang Johnson, 2004, p. 6). C'est donc en stigmatisant les différents parlers des paysans, ravalés au rang de dialectes, de patois, que l’État français est parvenu à poser les bases d'une politique de francisation. Ce qui est intéressant et apparaît au premier abord, après la lecture de cet ouvrage de Weber, c'est qu'on découvre l'existence de deux France qui vivaient côte à côte, l'une dans la «lumière », l'autre dans les «ténèbres» et qu'il faudrait soustraire à l'ignorance et à la «sauvagerie». En 1871, Gambetta disait des paysans qu'ils étaient « intellectuellement en retard de quelques siècles sur la partie éclairée du pays », que «la distance est énorme entre eux et nous [...], nous qui parlons notre langue, tandis que, chose cruelle à dire, tant de nos compatriotes ne font encore que la balbutier»(Weber, 1983, p. 20). Cette citation de Gambetta est plus que symptomatique du complexe de supériorité que les élites françaises éprouvaient à l'égard de leurs compatriotes paysans, qu’ils considéraient tout

1. Titre dont s'est inspirée Nancy Kwang Johnson pour un de ses articles présentés à l'Annual Meeting of the Canadian Political Science Association, 3-5 juin 2004 : «Senegalese "into Frenchmen"? The French technology of nationalism in Senegal». 
simplement comme incultes parce que ne parlant pas une langue porteuse de civilisation comme la langue française. Cette citation me paraît également intéressante en ce sens qu'elle est d'une ressemblance frappante avec celle de Vallon, alors gouverneur du Sénégal de 1889 à 1893. En effet, le contre-amiral Aristide Vallon affirmait ceci à l'égard des populations dont il avait la charge : "Nous sommes, vis-à-vis de ces peuplades, dans la même situation que visà-vis de nos paysans, nous leur devons l'instruction comme nous la devons à ces derniers. » (Kwang Johnson, 2004, p. 6)

À travers cette phrase, le projet colonial de la France était tout à fait clair. La France entendait mettre sur pied une politique d'assimilation à l'endroit de ses colonies africaines par le biais d'une instruction en français. Au même titre que les paysans français, les habitants de la Sénégambie allaient être francisés, et pour ce faire, la langue demeurait un élément incontournable aux yeux de l'autorité coloniale.

Cette politique d'assimilation a commencé avec la création des quatre communes (Saint-Louis, Rufisque, Dakar et Gorée) comme autant de territoires français d'outre-mer soumis au régime législatif et administratif de la métropole. Du point de vue de l'instruction, un certain nombre de mesures ont été prises, débouchant sur la création d'institutions scolaires. Les Français ont compris, à partir de l'expérience avec les paysans, l'importance de l'école dans la diffusion d'une langue à même de satisfaire les besoins communicationnels les plus immédiats et de participer à la formation intellectuelle des «indigènes ». C'est à cet égard que Louis-Jean Calvet affirme que «[...] l'école est [...] utilisée en conformité avec un projet idéologique qui vient s'insérer harmonieusement dans le développement de la superstructure linguistique du colonialisme naissant» (Calvet, 1974, p.71).

\section{L'école comme instrument d'assimilation}

En 1817 s'ouvre la première école française à Saint-Louis sous la direction d'un instituteur laïc du nom de Jean Dard. Celui-ci est confronté d'emblée à une tâche de grande ampleur car, quelque statut que la langue française puisse avoir sous ces contrées, elle demeurera toujours une langue étrangère pour les enfants sénégalais. C'est alors que Dard commence à s'interroger sur la méthode à suivre. Deux voies s'offrent à lui : privilégier des leçons de langue (grammaire) pour une bonne connaissance des bases du français, ou passer par la langue maternelle des enfants, le wolof, en vue d'une traduction. Il opte pour cette dernière, qui consiste à faire acquérir aux enfants une conscience linguistique claire de leur langue maternelle avant de les faire entrer dans une langue étrangère, le français. La voie choisie par Jean Dard, le détour par la langue maternelle des enfants, nécessite une bonne maîtrise du wolof. II l'apprend et le 
maîtrise au point d'en écrire une grammaire et un vocabulaire². Ses élèves peuvent ainsi lire en wolof pour ensuite traduire en français. En procédant de cette manière, Dard s'éloigne du fameux «projet méthodologique» (Calvet, 1974, p. 71) tracé par les autorités coloniales, qui d'ailleurs ne tardent pas à réagir. En 1822, Jean Dard est démis de ses fonctions d'enseignant sous le prétexte de son inefficacité pédagogique.

Restant persuadé du bien-fondé de son choix après sa destitution, Dard affirmait :

La civilisation des Ouolofs est plus que négligée, elle est mise à l'oubli puisqu'on a cessé d'instruire les noirs au Sénégal dans leur langue. Car quoi qu'on en dise, il faut que les noirs soient instruits dans leur langue maternelle, sans cela point d'établissements durables, point de civilisation [...] j'ai souvent remarqué que le jeune noir n'épelle les mots du français que dans le but d'en former des sons propres à son langage maternel. Si après les avoir étudiés, il ne retrouve qu'un son barbare à ses oreilles, il s'écrie : Kelley don dara (cela ne signifie rien) et ne veut plus étudier. Mais, si au contraire on lui donne à épeler un mot africain, alors il le répète jusqu'à ce qu'il puisse l'écrire sur le sable et l'expliquer à ses camarades. (Dard, 1826, p. 213)

Voilà un fait anecdotique très intéressant tant il est symptomatique de la politique obsessionnelle de francisation au Sénégal pendant la colonisation. Et Moussa Fall (2003, p. 152) de souligner, à propos de cette histoire, que «malheureusement, il [Dard] ignorait sans doute que derrière le projet d'instruction qu'on lui demandait d'appliquer, se cachait un objectif de glottophagie et de destruction culturelle inhérent à la politique d'assimilation que la France avait adoptée».

La méthode de Dard centrée sur la traduction a été jetée aux oubliettes, même si l'école mutuelle a continué de tourner car son but était, selon Denise Bouche (1968, p.112), de s'occuper « de l'enseignement de la lecture, de l'écriture et des quatre règles ». Le souci des autorités coloniales était de former des fonctionnaires subalternes tout juste capables de lire et d'écrire pour servir d'interprètes et faciliter le commerce avec les populations.

De 1841 à 1903, toujours d'après D. Bouche, les écoles publiques de garçons de Saint-Louis et de Gorée, puis celles de Rufisque et de Dakar ont été ouvertes et tenues par les Frères Plöermel. Mais avant eux, il y a eu les sœurs de Saint-Joseph-de-Cluny à Saint-Louis et Gorée ainsi que les Dames de l'Immaculée Conception de Castres à Dakar et Rufisque pour l'instruction des jeunes filles. Face à l'échec de la méthode de Dard, les autorités coloniales ont fait appel aux Frères Plöermel afin de « corriger la faiblesse de niveau » (Fall, 2003,

2. Dictionnaire français-wolof et français-bambara, suivi du dictionnaire wolof-français, Paris, Imprimerie royale, 1825. Grammaire Wolofe ou méthode pour étudier la langue des noirs en Sénégambie suivie d'un appendice, Paris, Imprimerie royale, 1826. 
p.153). Elles ont mis en place un système d'éducation calqué directement sur le modèle français sans tenir compte des réalités du pays. Leur méthode s'éloignait de celle de Dard dans la mesure où elle était axée sur l'enseignement traditionnel de la langue avec interdiction totale de parler les langues locales. Il faut rappeler, peut-être, que les frères avaient obtenu des autorités coloniales françaises qu'elles glissent dans leur contrat une clause exigeant d'enseigner le même programme qu'en métropole (Bouche, 1968).

\section{La guerre aux écoles coraniques}

En 1856, en butte à la méfiance de la population musulmane, le gouverneur Faidherbe avait créé l'école des otages 3 à Saint-Louis. Nombre de musulmans voyaient dans ces institutions des lieux de perdition et de conversion, les considérant comme un instrument de propagande au service du catholicisme, une instance prosélyte, d'autant que la plupart du temps, ces établissements étaient tenus par des hommes d'église. Cela constituait à leurs yeux un danger éminent pour la préservation de leur foi et de leur culture. Faidherbe, en bon administrateur, eut l'idée de laïciser cette institution nouvelle pour dissiper ces craintes, sans parvenir pour autant à endiguer une désaffection due à la concurrence des écoles coraniques. En 1857, à travers tout un arsenal juridique contraignant, il entra en conflit direct avec lesdites écoles. L'article 5 de son arrêté ${ }^{\circ} 6$ du 22 juin 1857 stipule que « les maîtres d'écoles musulmanes seront obligés de conduire ou d'envoyer tous les jours à la classe du soir (soit celle de l'école laïque, soit celle des Frères) tous les élèves de 12 ans ou audessus»4.

On voit bien l'ampleur des préoccupations des autorités coloniales à vouloir imposer coûte que coûte le français, et ce, par tous les moyens. En 1870, un autre arrêté du gouverneur (voir ci-après) va encore beaucoup plus loin dans la lutte contre la concurrence « déloyale» des écoles coraniques :

Nous gouverneur du Sénégal et dépendances [...] considérant que le but de l’administration de la colonie, en régularisant par ledit arrêté l'institution des écoles musulmanes, a été de rechercher à s'assimiler les enfants indigènes. Que ce but n’a pas été atteint jusqu'ici, par suite de l'indifférence apportée par les maîtres d'écoles. Attendu que le moyen le plus efficace d'arriver à ce résultat paraît être d'exiger désormais que ces maîtres habituent les enfants à comprendre et à parler la langue française. [...]

Article 1 : Nul ne pourra, à l'avenir obtenir une autorisation à tenir une école musulmane [...] s'il ne justifie savoir le français devant un jury [...]

3. Écoles créées par la France au Sénégal et que les fils de notables locaux étaient contraints de fréquenter poury devenir les auxiliaires de l'autorité coloniale.

4. Article 5 de l'arrêté nº 6 du 22 juin 1857 (Fall, 2003, p. 153). 
Article 3 : Les élèves qui suivent les écoles musulmanes devront y apprendre à parler le français. Ceux qui, au bout de deux ans, ne sauront pas se faire comprendre couramment dans cette langue, ne suivront plus lesdites écoles, et ne pourront plus fréquenter l'école des Frères ou l'école laïque. 5

Au-delà de cet arsenal juridique contre les écoles coraniques, une campagne a été orchestrée qui visait à déconsidérer la langue arabe et les musulmans, assimilés, à travers la plume et les discours d'un certain nombre d'administrateurs coloniaux, à des fanatiques cherchant à contrecarrer le projet colonial et, par là, les intérêts commerciaux de la France. Dès lors, le «musulman fanatique » incarnait l'ennemi à combattre. Et l'arme la plus efficace pour ce faire demeurait la langue française. Le gouverneur William Ponty affirmait, à propos des «vertus thérapeutiques» de la langue française contre le «fanatisme religieux des musulmans» : «[...] nul n'ignore en effet que l'étude du français est le remède le plus efficace qui puisse être opposé au fanatisme et l'expérience nous apprend que les musulmans qui connaissent notre langue sont moins imbus de préjugés que leurs coreligionnaires qui ne savent que l'arabe ${ }^{6}$.

Dans le combat pour la francisation, les langues locales ne faisaient pas seules l'objet de discrédit : la langue arabe était elle aussi considérée comme langue de désobéissance et de prétention. En présentant le français comme «remède» au fanatisme, était établi le pouvoir que les Français accordaient à leur langue.

\section{Senghor et les langues nationales : francisation ou Négritude?}

C'était en 1937. J'enseignais, alors, le français - avec les langues classiques depuis deux ans, au lycée Descartes de Tours. Venu passer les grandes vacances dans mon Sénégal natal, je fus sollicité de donner une conférence. J'avais choisi, comme thème, Le Problème culturel en AOF. La foule des évolués, blancs et noirs mêlés, s'écrasait dans la grande salle de la Chambre de commerce de Dakar. On s'attendait à m'entendre exalter la culture gréco-latine, du moins la culture française. Devant le Gouverneur général ébahi, je fis une charge vigoureuse contre l'assimilation et exaltai la Négritude, préconisant le «retour aux sources» : aux langues négro-africaines. Ce fut un succès de scandale, plus, au demeurant, chez les Africains que chez les Européens. «Maintenant qu'il a appris le latin et le grec, murmuraient ceux-là, il veut nous ramener au wolof.» (Senghor, 1962, p. 837)

Cette citation dénote l'ambiguïté de son auteur, Léopold Sédar Senghor, premier président de la République du Sénégal. En effet, elle permet de voir l'existence de deux Senghor : l'intellectuel engagé (idéaliste) et l'homme politique. On verra que le discours de ce dernier sur les langues varie en fonction

5. Arrêté no 85 du 28 février 1870 (Fall, 2003, p. 154).

6. Extrait de la circulaire no 82 c du 30 août 1910 relative à l'enseignement (Fall, 2003, p. 155). 
de ces deux positions. Lors de cette fameuse conférence, l'auteur a prétendu avoir défendu les langues locales au détriment de la langue française, mais il n'en demeure pas moins qu'il fut un fervent défenseur de la langue française et de la francisation. Pendant sa présidence (juste après l'Indépendance, en 1960), le français a continué à jouir de son prestige d'antan, cela pour, disaitil, consolider l'unité politique du pays :

Par quelle langue, si nous voulons préserver l'unité nationale? Et comment, alors qu'il n'y a même pas une bonne grammaire du wolof, enseigner les sciences modernes et réussir là où des langues écrites depuis mille ans essuient encore des échecs? (Senghor, 1983, p. 622)

Cette interrogation tempère l'ardeur un peu frondeuse de la première citation : si le jeune diplômé, fraîchement arrivé au pays, imbu des valeurs et principes de la Négritude, prône un temps le bilinguisme franco-sénégalais, il est vite rattrapé par la réalité et la complexité de la problématique de gestion des langues au Sénégal. Les déclarations d'intention à elles seules ne suffisent pas. Il faut prendre en compte d'autres facteurs, des facteurs qui ont contraint le président-poète à adopter une posture francophile et à faire son choix en fonction des intérêts de la Nation, et en fonction aussi d'un réalisme politique et économique. C'est ainsi que dans l'article 1 de la Constitution de 1963, le français est déclaré seule langue officielle du pays malgré l'avantage que la langue wolof marquait vis-à-vis des autres langues nationales. Il faut aussi noter que jamais présidence n'aura suscité autant de querelles linguistiques que celui du président-poète, et jamais président n'aura autant pensé, écrit et glosé sur la problématique des langues et de leur destin.

\section{La politique linguistique sous Senghor}

Cette absence de politique claire à l'égard des langues nationales n'a pas manqué de provoquer une guerre entre le président et ses opposants, surtout du côté des marxistes-léninistes dont les figures de proue restaient Cheikh Anta Diop, Pathé Diagne, Majmouth Diop et autres. Ces derniers, vaillants défenseurs des langues nationales et par ailleurs nationalistes, étaient pour la réhabilitation et l'introduction des langues nationales dans le circuit de l'enseignement formel et considéraient le français comme étant un cheval de Troie de l'impérialisme de l'ancien colonisateur. Ils étaient alors tous pour l'introduction des langues nationales dans l'enseignement, non pas pour faciliter l'enseignement de la langue française mais tout simplement pour lutter contre toute politique de francisation.

Senghor, dans la préface du livre de Pierre Dumont, justifie cette absence de volonté politique à l'égard des langues nationales par le volume et le nombre 
des réformes engagées par le gouvernement sénégalais à travers le triple défi que devait relever la nouvelle Nation : politique, économique et culturel.

Il reste qu'une fois obtenue l'indépendance, le chef de l'État et son gouvernement durent commencer par le commencement, comme j'aime à le dire. C'est ainsi qu'ils eurent à mener la lutte sur les trois fronts de la politique, de l'économie et de la culture pour surmonter deux tentatives de coups d'État, la détérioration des termes de l'échange, qui avait remplacé «l'économie de traite», enfin, la révolte étudiante de 1968... (Dumont, Préface, 1983, p.12)

Ce n'est qu'en 1971 qu'un décret7 a fait son apparition, portant sur la transcription des langues nationales, ce qui constituait « un premier pas important sur la voie d'une politique linguistique» (Hesseling, 1985, p. 353). Ce décret fixait la transcription des six langues nationales et ce, afin de les introduire dans l'enseignement formel. Néanmoins, cette tentative d'introduction des langues nationales dans l'enseignement s'est soldée par un échec pour des raisons diverses : par exemple, le refus des parents d'envoyer leurs enfants dans ces écoles, le manque criant de moyens financiers pour former des enseignants, etc. Pendant ce temps, les opposants restaient sur le pied de guerre, car ils voyaient en lui - les marxistes-léninistes surtout - un ennemi des langues et cultures africaines. Cheikh Anta Diop n'avait-il pas écrit quelques années auparavant, que «[...] l'unité linguistique sur la base d'une langue étrangère, sous quelque angle qu'on l'envisage, est un avortement culturel» ? (Diop, 2008, p. 25)

En effet, Senghor, agrégé de grammaire française, considérait les langues locales comme «des langues d'intuition » et la langue française comme « une langue de raisonnement», donc une langue d'enseignement et porteuse de civilisation. Il allait jusqu'à justifier le maintien de la langue française après l'Indépendance par sa clarté, sa concision et sa capacité à dire la modernité et la technique. Déjà, quelques années plus tôt, en 1962 plus précisément, il avait esquissé certaines raisons pour justifier le maintien de cette langue :

Troisième raison : la syntaxe. Parce que pourvu d'un vocabulaire abondant, grâce, en partie aux réserves du latin et du grec, le français est une langue concise. Par le même fait, c’est une langue précise et nuancée, donc claire. Il est, partant, une langue discursive, qui place chaque fait, chaque argument à sa place, sans en oublier un. Langue d'analyse, le français n'est pas moins langue de synthèse. On n'analyse pas sans synthétiser, on ne dénombre pas sans rassembler, on ne fait pas éclater la contradiction sans la dépasser. Si, du latin, le français n'a pas conservé toute la rigueur technique, il a hérité toute une série de mots-pierre d'angle, de mots-ciment, de mots-gonds. Mots-outils, les conjonctions et les locutions conjonctives lient une proposition à l'autre, une idée à l'autre, les subordonnant l'une à l'autre. Elles indiquent les étapes nécessaires de la pensée active : du

7. Décret n 71-566 du 21 mai 1971 relatif à la transcription des langues nationales, complété par le décret nº 72-702 du 16 juin 1972. 
raisonnement. À preuve que les intellectuels noirs ont dû emprunter ces outils au français pour vertébrer les langues vernaculaires. (Senghor, 1962, p. 839-840)

C'est ici l'évidence : le bilinguisme que le président Senghor appelle de ses vœux est un bilinguisme dominé par le français. Il est loin de ce bilinguisme équilibré qu'il prônait avant qu'il n'accédât à la magistrature suprême. Le français occupe la place centrale, car pour lui, les autres langues ne sont pas assez outillées, fixées pour pouvoir restituer l'âme de leur peuple. En tant que poète, il croit que les langues africaines doivent assurer leur part de poéticité pour pouvoir véhiculer les cultures ou civilisations de leur peuple à l'image de la langue française. II faut donc qu'il y ait des productions littéraires conséquentes pour que ces langues méritent d'être considérées comme des langues à part entière. Ce n'est qu'à partir de là qu'elles peuvent acquérir le statut de langues d'enseignement. Un enseignement tributaire de la richesse de leurs productions littéraires. C'est cette vision poétique et techniciste de la langue qui lui servait d'argument principal pour répondre à ses adversaires, partisans du wolof: "Comment [introduire la langue wolof dans l'enseignement], alors qu'il n'y a même pas une grammaire de la langue wolof?» (Senghor, 1983, p. 622)

On voit bien que Senghor convoquait, à tort ou à raison, les idées de la politique assimilationniste chère à l'ancien colonisateur ardemment combattu, et qui consistaient à voir les langues africaines comme des «patois», des parlers, des dialectes enkystés dans le royaume de l'oralité, donc irrationnels et incapables de se mêler au logos; des langues dont les Africains devaient se débarrasser au profit d'une langue écrite moderne porteuse de civilisation, de culture et des valeurs de la modernité : la langue française.

Une telle conception de la langue ne peut que valoriser le rôle du français, lequel semble déjà avoir tous les prérequis établis par Senghor.

Tout d'abord remplacer le français, comme langue officielle et comme langue d'enseignement, n'est ni souhaitable, ni possible. Si du moins nous ne voulons pas être en retard au rendez-vous de l'An 2000. En effet, il nous faudrait au moins deux générations pour faire d'une de nos langues nationales un instrument efficace pour l'enseignement des sciences et des techniques. Et à condition que nous en eussions les moyens financiers et humains, c'est-à-dire des savants et des techniciens assez qualifiés. Or, en cette seconde moitié du xx $x^{e}$ siècle, quarante à cinquante ans de retard, cela ne se rattrape pas. (Cité par Dumont, 1983, p. 207)

\section{La bataille des langues : Senghor et les nationalistes}

La mise en équation des langues consistant à dire qu'une vraie langue équivaut à une langue de littérature a provoqué des réactions chez les défenseurs des langues nationales. Dans le but de prouver qu'elles peuvent, au même titre que les langues occidentales, porter des valeurs de civilisation, les défenseurs des 
langues nationales sont tombés dans le piège de Senghor. Tout comme lui, ils ont utilisé la rhétorique et les stratégies consistant à prôner le repli sur l'unité, l'homogène aboutissant au nationalisme. C'est à partir de ce moment que des travaux sur la langue wolof ont été entamés dans le dessein de revitaliser les langues nationales: Cheikh Anta Diop, « doyen des hommes de lettres wolof » pour parler comme O'Brien, a traduit en wolof La théorie de la relativité d'Einstein et Le manifeste du parti communiste ainsi que des œuvres de la littérature française (O’Brien, 2002, p.150-151). Au même moment, le professeur Sakhir Thiam publiait des manuels de mathématiques en wolof. Afin de pouvoir mener à bien ce combat contre la tentative verticale d'imposition de la langue française, la plupart de ces nationalistes s'étaient organisés à travers des organes de presse éditant en langues nationales. C'est ainsi que Cheikh Anta Diop, chef du RND 8 , a créé Siggì, un journal franco-wolof, et que de son côté, l'écrivain et cinéaste Sembène Ousmane a créé Kaddu10. Ces organes de presse ont été des instruments efficaces contre la politique de Senghor. Ils ont joué le rôle d'espaces au sein desquels on rendait compte du génie du peuple wolof. C'est ainsi qu'en $1975^{11}$, un décret relatif à l'orthographe et à la séparation des mots en wolof a été publié par le gouvernement de Senghor dans le dessein de faire obstacle à ses adversaires politiques. Le rôle de ce décret portant sur la gémination consistait à interdire l'utilisation des doubles consonnes dans les textes wolof (notamment dans les journaux en wolof), alors que dans cette langue, la gémination « est un phénomène phonologiquement attesté», donc susceptible «d'être graphiquement marqué par un redoublement de la note phonique concernée » (Cissé, 2005). Toujours pour provoquer ses adversaires nationalistes, Senghor a fait voter le 10 octobre 1977 une autre loi importante pour une régulation orthographique des langues nationales, en invoquant les raisons suivantes :

À l'heure où une littérature et une presse en langue nationale prennent leur essor dans notre pays pour la première fois d'une manière significative, il est impossible pour les pouvoirs publics de tolérer que s'instaurent dans ce domaine si sensible de la langue, l'anarchie et la confusion et que l'on assiste à l'éclosion et la profusion de système «sauvage» de transcription des langues nationales ne relevant que de l'individualisme de leurs auteurs et de l'improvisation. ${ }^{12}$

Il semble, si on en croit O'Brien, que l'abbé Boilat13 se soit toujours élevé contre l'utilisation des doubles consonnes dans la transcription des langues

8. Rassemblement national démocratique.

9. Siggi signifie en wolof «être debout, se redresser».

10. Kaddu signifie en wolof «parole».

11. Décret $n^{0} 75$-1026 relatif à l'orthographe et à la séparation des mots en wolof.

12. Loi nº 77-55 du 10 avril 1977 relative à l'application de la réglementation en matière de transcription des langues nationales.

13. L'abbé Boilat est né le 23 avril 1814 à Saint-Louis du Sénégal d'un père français et d'une mère signare. Il a été envoyé en France par des missionnaires pour faire des études afin de devenir enseignant au Sénégal. Il a été ordonné prêtre en 1841 et, à son retour du Sénégal, il s’est vu 
nationales et que par conséquent, "c'est de son autorité que s'est réclamé L. S. Senghor, ce Jules Ferry sénégalais qui menace les élèves récalcitrants de la règle du maître " (O'Brien, 2002, p. 153). Mais quelle que soit l'origine de cette idée, les premiers visés restaient incontestablement les défenseurs du wolof et des autres langues nationales qui utilisaient la gémination. En témoignent les noms de leurs journaux : Siggi, Kaddu. Ils sont d'autant plus attaqués que cette loi est renforcée par une autre, en 1977 ${ }^{14}$, prévoyant de lourdes sanctions aux contrevenants : «... trois mois de détention ou une amende pouvant aller jusqu'à un million de francs CFA... ». (O'Brien, 2002, p.153). La question sur les langues devenait ainsi un problème politique. Certains chercheurs comme Cissé (2005) ont été jusqu'à dire que Senghor utilisait les questions linguistiques pour régler des contentieux politiques. Selon Cissé (2005), le président avait «stratégiquement orienté le débat sur la politique linguistique vers des apories », transformant un problème scientifique du ressort des linguistes - la graphie et le découpage des langues - en une affaire politique. Mais une forte résistance s'est dressée contre le «président-linguiste» car la communauté scientifique s'est rangée du côté des nationalistes.

En 1978, la Constitution sénégalaise de 1963 fut encore révisée. Neuf articles furent modifiés, dont un portant sur la langue. Selon Hesseling (1985), Senghor en profita pour « définir constitutionnellement la politique linguistique du pays». Désormais, dans l'article 1 de la Constitution, où il était stipulé que le français était la langue officielle, on reconnaissait six «langues vernaculaires » comme langues nationales. Elles étaient déclinées par ordre alphabétique en signe de neutralité (le diola, le malinké, le poular, le soninké, le sérère et le wolof). Elles acquéraient par là un statut «officiel», même si le concept de «langues nationales» restait flou, faisant dire à certains linguistes que leur statut demeurait purement symbolique.

Il serait abusif, dès lors, de parler de la mise en place, au cours du mandat présidentiel de Senghor, d'une politique linguistique équitable. Le président avait préféré adopter une position attentiste. À travers sa vision culturaliste et techniciste de la langue, il attendait que les langues nationales fussent susceptibles de mériter le statut de langue à part entière, ce qui n'avait fait que conforter le privilège accordé à la langue française. Par conséquent, ont été produits un certain nombre de discours (politiques, linguistiques, juridiques) visant à défendre, légitimer et institutionnaliser la présence de la langue française. C'est ce qu'on appelle la francisation : la création des conditions techniques

confier la direction de l'enseignement sous le mandat du gouverneur Bouët Willaumetz. C'est ainsi qu'il a créé un établissement secondaire, mais a fini par se retirer pour cause de difficultés avec le personnel enseignant. C'est un des premiers à avoir écrit sur le Sénégal. On lui doit Esquisses sénégalaises, une étude sur l'histoire du Sénégal avec ses propres illustrations.

14. Loi no 77-55 du 28 mars 1977 instituant des sanctions administratives et des poursuites judiciaires en cas de non-respect des règles édictées en matière de transcription. 
et matérielles d'instauration et de renforcement de la langue française en vue de sa propagation. Elle a conduit, par effet de résistance, à d'autres formes d’homogénéisation.

À travers cet article, nous avons essayé de passer en revue les différentes étapes de la vie politique du Sénégal sous le prisme de la question linguistique, de la colonisation jusqu'aux années quatre-vingt. Les politiques linguistiques et éducatives mises en place pendant la colonisation ont été favorables à l'imposition de la langue française. Cette imposition s'est faite par le biais de l'éducation, avec l'ouverture des premières écoles françaises. Malgré tous les dispositifs technologiques et juridiques mis en place pour la francisation, le poids de la religion, l'exode rural, l'urbanisation et d'autres dynamiques sociales ont participé à l'émergence et à l'expansion du wolof comme langue véhiculaire entre différentes communautés rassemblées dans les centres urbains. Pour autant, la langue wolof, même avec l'avènement de l'indépendance, n'a pas bénéficié d'un statut officiel. Les tentatives de politiques linguistiques postcoloniales en faveur des langues nationales ont toutes échoué par manque de moyens. Le rapport de force était en faveur de la langue française, langue de l'administration, de l'école et de l'armée, ce qui a entraîné l'émergence de mouvements nationalistes en faveur de la langue wolof et des langues nationales.

\section{Références}

ARACIL Lluis Vicent, 1982 [1965], "Conflit linguistique dans l'Europe moderne», Nancy, Centre européen universitaire, dans Papers de sociolingüistica, Barcelone, La Magrana, p. 23-28.

Bouche Denise, 1968, "Autrefois notre pays s'appelait la Gaule... Remarques sur l'adaptation de l'enseignement au Sénégal de 1817 à 1960 », Cahiers d'études africaines, vol.VIII, n² 29, p.110-122.

CAlvet Louis-Jean, 1974, Linguistique et colonialisme. Petit traité de glottophagie, Paris, Payot.

CISSÉ Mamadou, 2005, "Langues, État et société au Sénégal», SudLangues, en ligne : www.sudlangues.sn/IMG/pdf/doc-109.pdf (consulté le 1er juillet 2014).

— 2007, «De l'assimilation à l'appropriation : essai de glottopolitique senghorienne», SudLangues, $n^{\circ}$ 7, en ligne : www.sudlangues.sn/IMG/pdf/doc-171.pdf (consulté le $1^{\text {er juillet 2014) }}$

DARD Jean, 1826, Grammaire wolofe ou méthode pour étudier la langue des Noirs qui habitent les royaumes du Bourba Jolof, du Walo, de Damel, de Bour Sine, de Saloum, de Sénégambie, Paris, Imprimerie royale.

Dıop Cheikh Anta, 2008, Fondements économiques et culturels d'un État fédéral d'Afrique Noire, Paris, Présence africaine.

Dıouf Makhtar, 1994, Sénégal. Les ethnies et la Nation, Paris, L’Harmattan. 
Dumont Pierre, 1983, Le français et les langues africaines, Paris, ACCT-Karthala.

FALL Moussa, 2003, "Baisse du niveau des élèves en français : mythe ou réalité. Le cas du Sénégal», SudLangues, nº 3, p.150-161.

GUESPIN Louis, MARCELLESI Jean-Baptiste, 1986, «Pour la glottopolitique », Langages, vol.XXI, n०83, p.5-34.

Hesseling Gerti, 1985, Histoire politique du Sénégal. Institutions, droit et société, Paris, Karthala.

KWANG Johnson Nancy, 2004, "Senegalese “into Frenchmen”? The French technology of nationalism in Senegal », Annual Meeting of the Canadian Political Science Association, 3-5 juin 2004, Canada, Nationalism and Ethnic Politics, vol. X, nº 1, p. 135-158.

NDAo Papa Alioune, 1996, Contacts de langues au Sénégal, étude du code-switching wolof/français en milieu urbain. Approches linguistiques, sociolinguistique et pragmatique, thèse de doctorat, Université Cheikh Anta Diop de Dakar.

- 2011, «Politiques linguistiques et gestions de la diversité linguistique au Sénégal : aspects sociolinguistiques", Revue de sociolinguistique en ligne, $\mathrm{n}^{\circ}$ 18. http:// www.univ-rouen.fr/dyalang/glottopol/telecharger/numero_18/gpl18_01ndao.pdf.

NDIAYE Daouda, 2008, L'enseignement des langues nationales à l'école primaire, le cas du wolofau Sénégal, thèse en science de l'éducation, Université Paris 8 Vincennes - Saint-Denis.

O'BRIEN D. Cruise, 1998, «The shadow-politics of wolofisation», The Journal of Modern African Studies, vol. XXXVI, $\mathrm{n}^{\circ} 1$.

O'Brien D. Cruise, Dıop Momar C., Dıouf Mamadou, 2002, La construction de l'État du Sénégal, Paris, Khartala.

PRInZ Manfred, 1996, L'alphabétisation au Sénégal, Paris, L'Harmattan.

SENGHOR Leopold S., 1962, "Le français, langue de culture », Esprit, n 11, Le français, langue vivante, p. 837-844.

- 1983, Liberté, t. IV, Socialisme et planification, Paris, Le Seuil.

WEB ER Eugen, 1983, La fin des terroirs. La modernisation de la France rurale, 1870-1914, Paris, Fayard. 
